\title{
Respiratory monitoring using fibre long period grating sensors
}

\author{
T. Allsop, R. Revees, D.J. Webb, I. Bennion \\ Photonics Research Group, Aston University, Aston Triangle, Birmingham, B4 7ET, U.K. \\ t.d.p.allsop@aston.ac.uk \\ T. Earthrowl, B. Jones \\ Clinical Biomedical Engineering Research Group, Aston University, Aston Triangle, Birmingham, \\ B4 7ET, U.K. \\ M. Miller \\ Respiratory Medicine, University of Birmingham, Birmingham, UK

\begin{abstract}
We demonstrate the use of a series of in-line fibre long period grating curvature sensors on a garment, used to monitor the thoracic and abdominal volumetric tidal movements of a human subject. These results are used to obtain volumetric tidal changes of the human torso showing reasonable agreement with a spirometer used simultaneously to record the volume at the mouth during breathing. The curvature sensors are based upon long period gratings written in a progressive three layered fibre that are insensitive to refractive index changes. The sensor platform consists of the long period grating laid upon a carbon fibre ribbon, which is encapsulated in a low temperature curing silicone rubber.
\end{abstract}

Keywords: Curvature Sensing, Long Period Gratings, Respiratory Monitoring

\section{INTRODUCTION}

Non-invasive measurement of thoracoabdominal surface motion can provide a comprehensive appreciation of respiratory function and enhance our understanding of respiratory physiology. Knowledge of the total and regional deflection of the surface during breathing not only provides a key to the volumetric flow of gases but also provides an insight into the thoracic and abdominal contributions and the recruitment of muscles. Research in this field has been predominantly laboratory based, the provision of a reliable quantitative clinical tool for making such measurements in ambulatory patients would advance routine respiratory function monitoring.

Konno and Mead [1] first demonstrated that respiratory gas flow could be measured at the surface of the chest and abdomen thus negating the need for measurement of flow at the mouth, which is the clinical standard at present. A number of measurement devices have been developed following Konno and Mead; techniques include: Respiratory Inductive Plethysmography (RIP) [2], Optical Reflectance Plethysmography (ORP) [3] and the use of magnetometers [4]. Some of the problems associated with the fore-mentioned techniques are that they rely heavily on model based signal processing, that the cost and realisation of a sufficiently dense array of sensors is prohibitive, that an off body referencing is required to track specified anatomical location, that in the case of RIP there is limited accuracy over wide variations in tidal volume and changes in posture after calibration [5] and that generally the instrumentation is bulky and has to be operated while the patient occupies a confined area. It follows that the technique is not suitable for routine ambulatory monitoring. The fundamental requirement here is to create a highly compliant sensor with which to track selected anatomical positions on the chest and abdomen surface.

This paper presents a possible solution to mobile respiratory function monitoring, using a sensor that utilises an optical fibre Long Period Grating (LPG) sensing element [6,7]. The curvature sensors are adhered to a garment, which is used to detect the shape/curvature variations of the chest and abdomen regions of a human torso during respiration. These results are used to obtain volumetric tidal changes of the human torso showing reasonable agreement with a spirometer used simultaneously to record the volume at the mouth during breathing. The sensors are also used to reconstruct the shape of a simulated human torso (a resuscitation manikin) during inflation

Novel Optical Instrumentation for Biomedical Applications II, edited by Christian D. Depeursinge,

Proc. of SPIE-OSA Biomedical Optics, SPIE Vol. 5864 (C) 2005 SPIE and OSA · 1605-7422/05/\$15 


\section{THE CURVATURE SENSOR}

The curvature sensor utilises an LPG as the sensing element. LPGs are photoinduced fibre devices that couple light from the core of a single-mode optical fibre into the cladding at discrete wavelengths and thereby generate attenuation bands in the transmission spectrum of the optical fibre core. LPGs are sensitive to strain $(\varepsilon)$, temperature $(T)$, and the surrounding refractive index $\left(n_{s}\right)$ [7] and LPGs have also been used as shape/bending sensors [8]; the LPG's sensitivity to these parameters can manifest itself in two ways: firstly the central wavelength of the attenuation band can shift in the spectral domain and secondly a change in the spectral transmission profile of the attenuation band may occur. The spectral shift of the attenuation band arises from the phase matching condition of the LPG, given by

$$
\lambda_{i}=\delta n_{e f f} \Lambda \equiv\left[n_{c o}\left(\lambda_{1}, n_{1}, n_{2}, T, \mu \varepsilon, R\right)-n_{c l} l v\left(\lambda_{i}, n_{1}, n_{2}, T, \mu \varepsilon, n_{s}, R\right)\right] \Lambda
$$

where $n_{c o}$ is the effective index of the core mode and $n_{c l}^{1 v}$ is the effective index of the $1 v^{\text {th }}$ radial cladding mode, both indices being dependent on the core refractive index $n_{1}$, the inner cladding refractive index $n_{2}$ and the wavelength $\lambda$. Also, $n_{c l}{ }^{l v}$ is a function of the refractive indices of the surrounding medium, $n_{s} . \Lambda$ is the period of the LPG, $T$ the temperature, $\varepsilon$ the strain and $R$ the curvature experienced by the fibre. Whilst equation 1 gives the spectral position of the attenuation band, the magnitude of the spectral shift induced by the measurands $\left(\varepsilon, T, n_{s}\right)$ is dependent upon two factors: the difference between the effective refractive indices and also the difference between the group effective refractive indices of the two modes [9]. The spectral sensitivity of LPGs to bending arises from two major components, the strain sensitivity $(\varepsilon)$ and the effective change in the refractive index profile of the fibre induced by the bend itself; the sensitivity can be calculated using a conformal mapping technique [10]. The bending of the optical fibre induces changes to the propagation constants of the cladding modes thus changing the group refractive indices as well as the effective refractive indices.

The LPG (length of $\sim 7 \mathrm{~cm}$ ) is written in a progressive three layered (PTL) fibre, which is inscribed using the point by point method [11]. The result of using such a fibre, is that the LPG produces attenuation bands that are insensitive to changes in the surrounding medium's refractive index. The LPG is laid upon a carbon fibre ribbon, with this then encapsulated in a low temperature $\left(\sim 90^{\circ} \mathrm{C}\right)$ curing silicone rubber, see figure 1 . This type of silicone rubber is used to minimise any drift in the position of the LPG's attenuation bands due to thermal annealing and to provide a thermally insulating layer for the respiratory function monitoring application (reducing the effects of rapid ambient temperature fluctuations). By incorporating the carbon fibre ribon, the sensor was designed to prevent the fibre from experiencing significant axial strain and yet still be highly flexible.

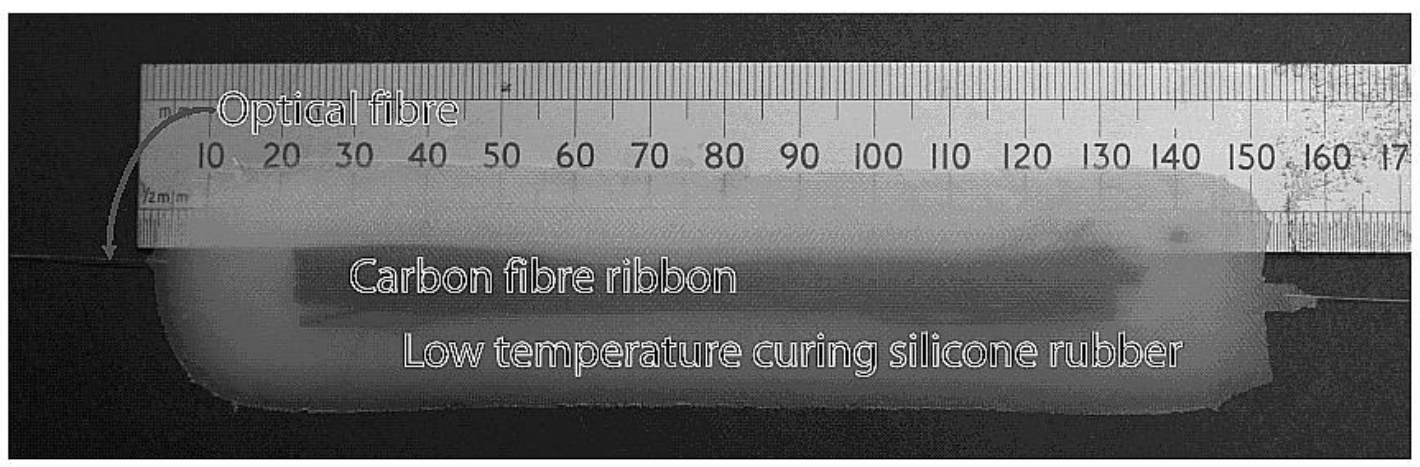

Figure 1. The curvature sensor 


\section{MONITORING HUMAN RESPIRATION USING CURVATURE SENSORS}

Four characterised curvature sensors (length $15 \mathrm{~cm}$ ) were connected in a series configuration. The sensor array was stitched to a Lycra vest for a practical evaluation of the sensors' suitability for this application. The curvature sensors had similar curvature resolutions, approximately $\sim 3.5 \times 10^{-2} \mathrm{~m}^{-1}$, with spectral sensitivities of $\frac{d \lambda}{d R} \approx 8 \mathrm{~nm} \mathrm{~m}$. The Lycra vest was placed on a human male torso at two different sets of locations: at the front and side. The sensors were illuminated with a broadband light source and the wavelength shifts of the attenuation bands were monitored with an OSA (sweep time $20 \mathrm{~ms}$, spectral resolution $0.1 \mathrm{~nm}$ ) which was controlled by a computer, see figure 2 .
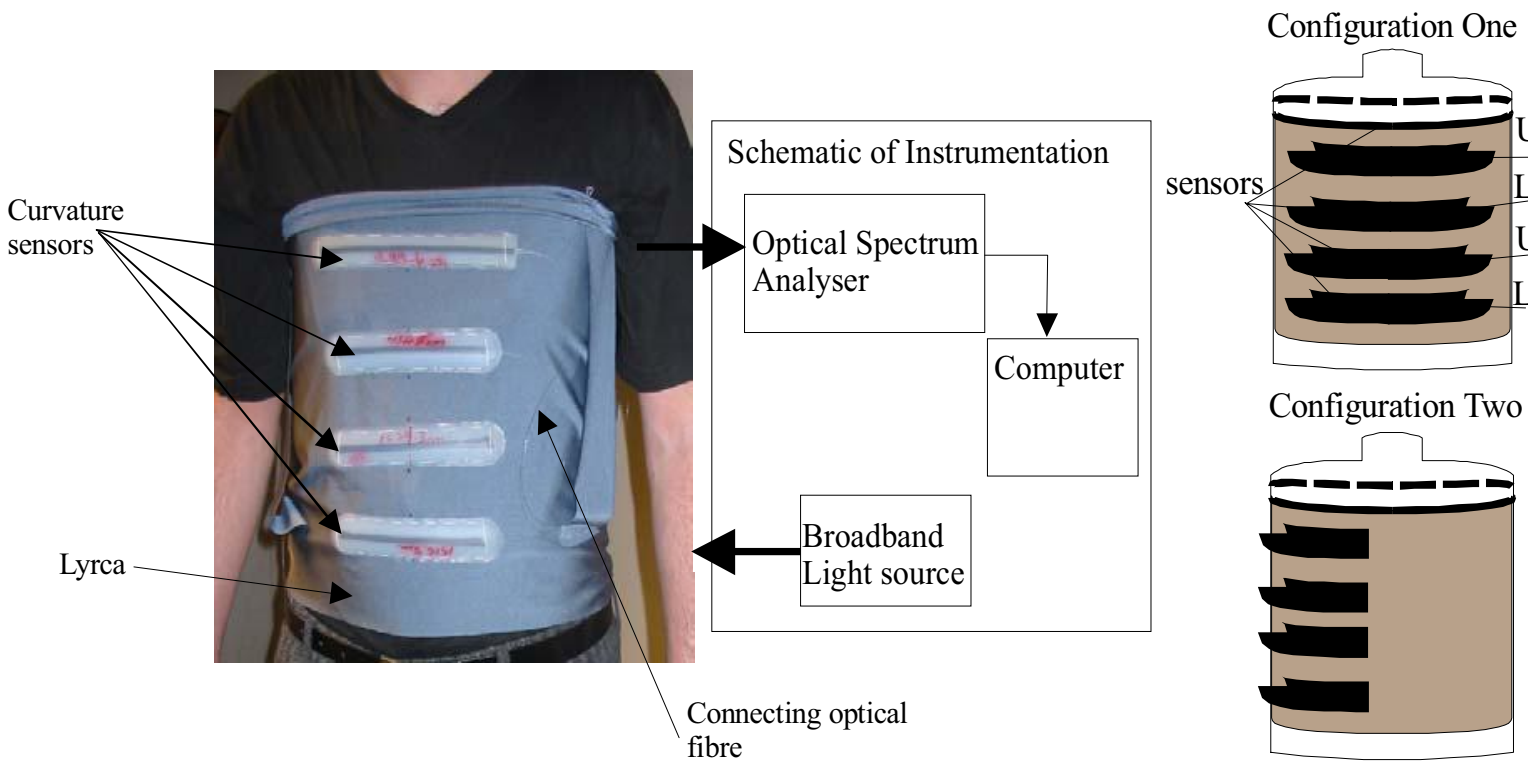

Figure 2. Schematic of optical instrumentation used for the monitoring of the curvature sensors.

The sensor array monitored, via the changes in curvature of the sensors, the thorax movement of a subject during respiration. Two examples of the curvature variation of the sensors during long tidal breaths are shown in figure 3 for two locations on the torso.
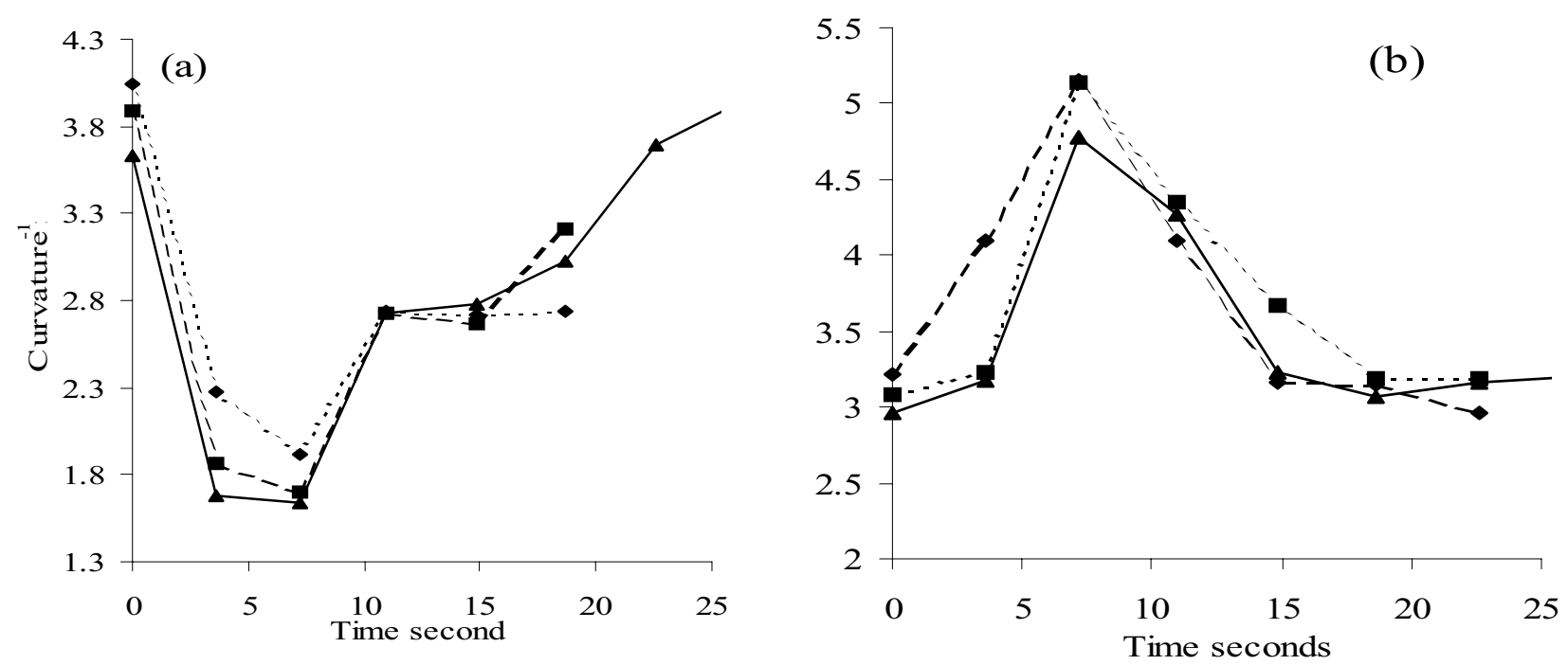

Figure 3. Results from a single sensor (a) at the lower torso for three breaths as function of time, the array location on the front of the torso. (b) at the upper abdomen for three breaths as function of time, the array location on the side of the torso. 
Inspecting figure 3 there are variations in curvature between each long tidal breaths but this is expected due to the difficulty in exactly repeating a given breathing pattern. It is noticeable that the general response is the same for each breath and this was found for all the sensors at the various locations on the thorax. Also the sensors can be used to distinguish between the various geometric variations associated with different locations on the thorax and abdomen during respiratory movement.

Whilst the sensors were being monitored during inspiration and expiration, the volumes were simultaneously recorded at the mouth of the subject using a turbine transduction spirometer system (Micro Medical, UK). Linear regression was applied to predict volume exhaled at the mouth from the sensor signals for the two spatial locations of the sensor array. It can be shown that from a simple system of these sensors an accurate assessment of tidal breathing can be made [12], which is similar to the precision obtained with an external optical system using 80 sensor points on the thorax [13]. A direct comparison is shown in figure 4 of the volume measured from the mouth with a spirometer and the volume calculated from the curvature-sensing array. A control of the sensors was also conducted; this was achieved by the subject holding their breath while several measurements were recorded. Using the calibrations already obtained for the sensors, the practical resolution of the sensors on the subject was found to be very similar to the resolutions obtained during initial characterisation of the sensors on the laboratory bench.

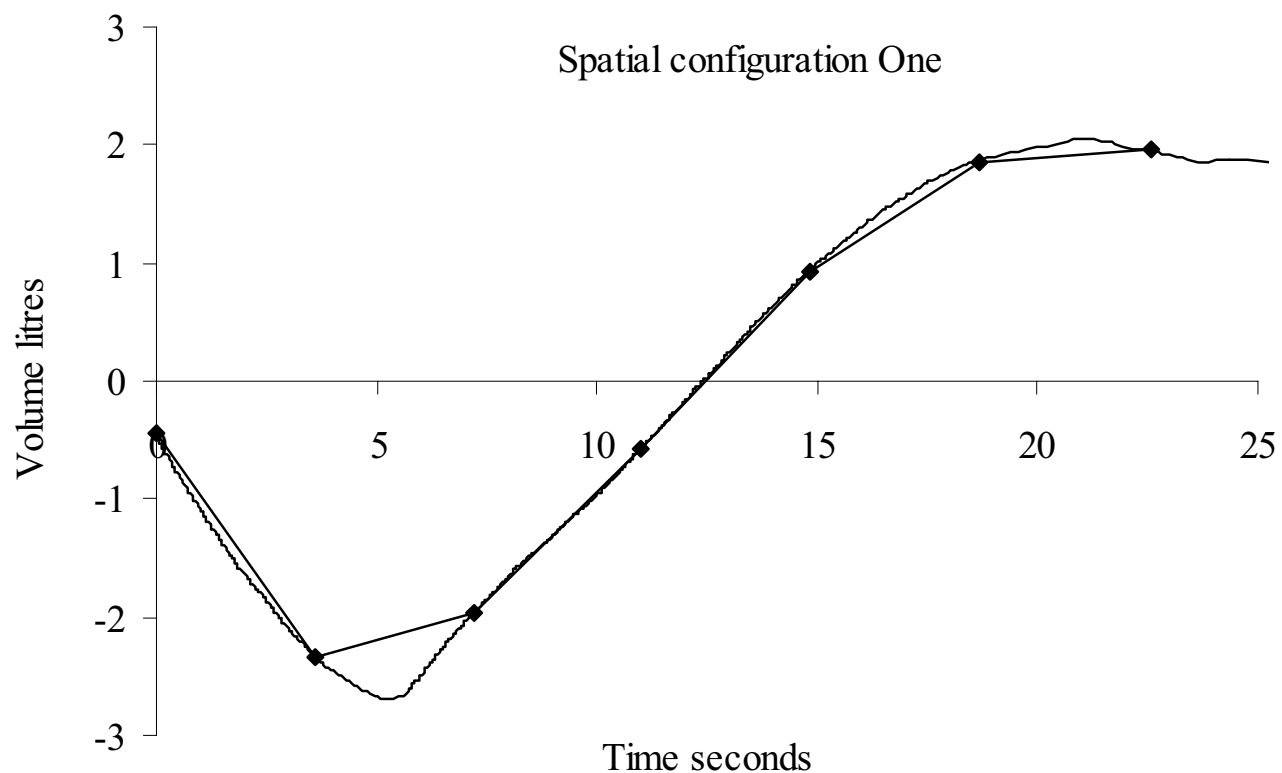

Figure 4. A typical volumetric variation during a long tidal breath. The measured volume from the spirometer and the calculated volume from the curvature sensors are shown as a function of time

\section{SHAPE RECONSTRUCTION}

To further demonstrate the utility of this approach in a more repeatable fashion, two in-line curvature sensors were used to record the variation of the curvature of a resuscitation training manikin during inflation from 0 to 0.9 litres in increments of 0.3 litres. Two locations were simultaneously monitored over the volume range then the sensors were moved to other locations and the process repeated for the same inflation volumes.

A derivative spectroscopy interrogation technique was used to detect the changes in the spectral transmission profile of the LPG's stop band induced by the bending of the LPG. Modulating the source wavelength (a narrow linewidth source such as distributed feedback (DFB) laser) at a given frequency will generate a series of harmonics of the modulating frequency [14] in the output signal transmitted by the LPG. It has been shown that the in-phase component of the $\mathrm{n}^{\text {th }}$ harmonic output is proportional to the $\mathrm{n}^{\text {th }}$ derivative of the spectral profile under investigation [15]. The amplitudes of the first and second harmonics are therefore proportional to the first and second derivatives of the spectral transmission function of the sensor, and the utility of our approach relies on the ratio of those derivatives being a unique 
function of the position in the spectral profile as well as being independent of any attenuation in the system. This interrogation technique has previously been reported with a single LPG fibre-grating device using a pigtailed DFB laser as the light source [14].

A total of 22 sensing positions were covered in all. The data obtained from the curvature were used to reconstruct the shape of the manikin's torso. The locations of the sensors are shown in figure 4a and the resulting reconstruction from the sensors is shown in figure 5b. A simple method to visualise the surface data in its reconstructed form was realised with commercial solid modelling software (SolidWorks) [16]. The surface depicted in figure $5 \mathrm{~b}$ was constructed using curvature data and triangulating the measured curvatures in their original (deflated) planes of orientation. The overall profile is determined by successively linking the arcs of curvature in those respective planes. Smoothing of the profile is accomplished by setting peripheral relationships between curves and the application of lofting algorithms built into the software At each discrete state of inflation the corresponding change of curvature at each location can be superimposed to represent motion of the manikin surface.

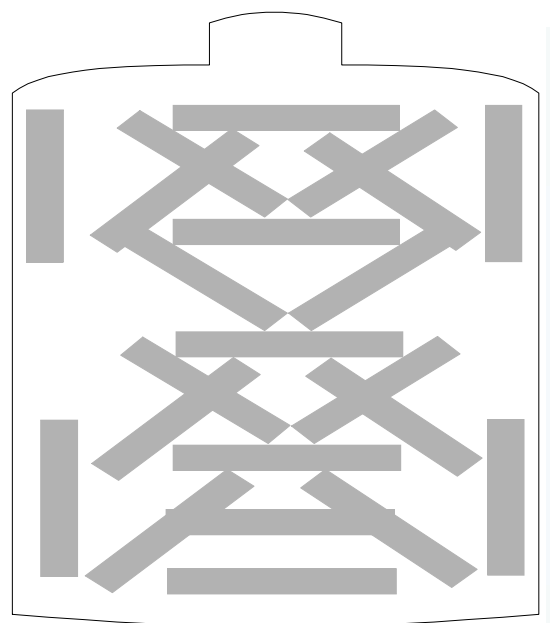

Figure 5a. Experimental sensor layout

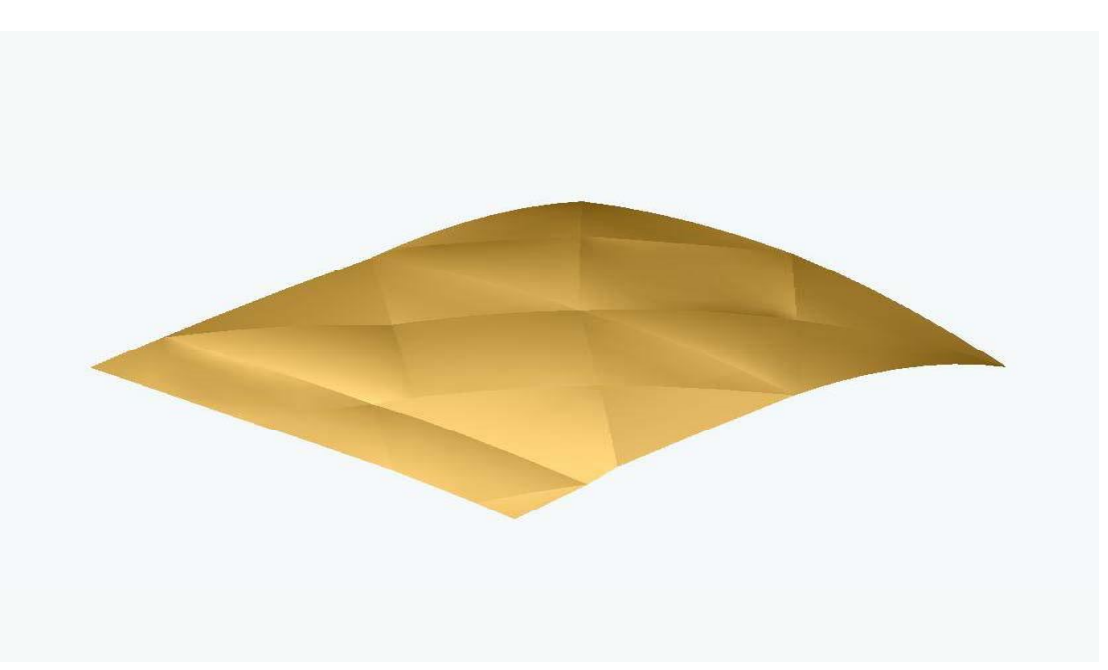

Figure 5b. Surface constructed from measured curvature data

\section{CONCLUSION}

A series of in-line curvature sensors on a garment have been used to monitor the thoracic and abdominal movements of a human during respiration. These results are used to obtain volumetric tidal changes of the human torso which showed agreement with a spirometer used simultaneously to recorded the inspired and expired volume at the mouth. It has also been demonstrated that such sensors can be used to distinguish between the various geometric variations associated with different locations on the thorax and abdomen during respiratory movement. It was also observed that the temporal evolution of the curvatures for a given torso location is repeatable on a breath-to-breath basis. This respiratory system has the potential to be made small, portable and cost effective, thereby permitting ambulatory monitoring of breathing.

\section{ACKNOWLEDGMENTS}

The authors would like to thank Fibrecore Limited, in particular Tim Hart, for supplying the PTL fibre and its specification and AEROVAC Systems Ltd for help in the development of the sensor platform, with special thanks to Matthew Fisher and Kevin Crowther. The work was funded by the UK Engineering and Physical Sciences Research Council and the Wellcome Trust (grant number 074649/Z/04/Z). 


\section{REFERENCES}

1. K. Konno, et al, J. "Measurement of the separate volume changes of the rib cage and abdomen during breathing", J. Appl. Physiol., vol.22, no.3, pp.407-422, 1967.

2. Watson H. The technology of respiratory inductance plethysmography. In ISAM 1979. Proceedings of the third international symposium on ambulatory monitoring. ED. Scott, FD. Rafferty, EB. \& Goulding, L., PUB. London: Academic, pp.537, 1980

3. SJ. Cala, et al, "Chest wall and lung volume estimation by optical reflectance motion analysis", J. Appl. Physiol.; vol. 8., no.6, pp.2680 2689,1996.

4. Levine S,et al, "Use of triaxial magnetometer for respiratory measurements", Jnl. Appl. Physiol.; vol.70, no.5, pp.2311-2321, 1991

5. R. B. Banzett, et al, “A simple reliable method to calibrate magnetometers and respitrace”, Jnl. Appl. Physiol., vol.79, no.6, pp.2169-2176, 1995

6. T. Allsop et al," A comparison and characteristics of sensing parameters of long period gratings written in three different types of fiber", Optical Fiber Technology vol.9, no.4, pp.210-223, 2003

7. V. Bhatia, "Applications of long-period gratings to single and multi-parameter sensing", Opt. Express, vol. 4, no. 11, pp.457-466, 1999.

8. H.J. Patrick, et al, "Long period fibre gratings for structural bend sensing", Electron. Letts., vol. 34, no. 18, pp. 1773-1775, 1998.

9. V. Bhatia, Properties and Sensing applications of long-period gratings $\mathrm{PhD}$ Thesis, Virgina Polytechnic Institute and State University, Blackburg, Virgina, 1996.

10. M. Heiblum, J.H. Harris, "Analysis of curved optical waveguides by conformal transformation”, Quant. Electron., vol.QE-11, no.2, pp. 75-83, 1975.

11. L. Zhang et al, "Design and realization of long-period grating devices in conventional and high birefringence fibers and their novel applications as fiber-optic load sensors", Selected Topics Quant. Electron., vol.5, no.5, pp. 1373$1378,1999$.

12 T Allsop, et al, "Embedded progressive-three-layered fiber long-period gratings for respiratory monitoring", J. Biomed. Opt. 8 (3), pp. 552-558, 2003.

13 Alverti A, et al, "Optoelectronic plethysmography in intensive care patients", Am J Resp Crit Med., vol. 161, pp.1546-1552, 2000.

14 P. Kluczynski, 2001, "Wavelength modulation absorption spectrometry - an extensive scrutiny of the generation of signals" Spectrochimica Acta. B, Vol 56, no.8, pp1277-1354.

15. T. Allsop, R. Reeves, R. Neal, I. Bennion, 2002, "A high accuracy/sensitive refractive index sensing system based upon a long period grating Mach-Zehnder interferometer with a heterodyne detection technique", Review Sci. Instr. vol.73, no.4, pp1702-1705.

16. SolidWorks Office Professional, SolidWorks Corporation, "http://www.solidworks.com/" 